\title{
Genderverteilung der Lehrenden in den Fächern Komposition, Elektroakustische Komposition und Musiktheorie an deutschen Hochschulen
}

\author{
Eine statistische Recherche
}

Irene Kletschke, Kirsten Reese

Schlagworte/Keywords: appointment policy; Berufungspolitik; Diversität; diversity; equality; female composers; female teachers; gender; Gleichstellung; Komponistinnen; weibliche Lehrende

Im Februar 2019 wurde von der Frauenbeauftragten der Universität der Künste (UdK) Berlin, unterstützt von Fachkolleginnen der Fakultät Musik und von klangzeitort, dem gemeinsamen Institut für Neue Musik der UdK Berlin und der Hochschule für Musik Hanns Eisler (HfM) Berlin, eine Recherche zur Genderverteilung der Lehrenden in den Fächern Komposition und Musiktheorie an Musikhochschulen in Deutschland in Auftrag gegeben. In einer Vorrecherche waren zunächst die auf den Webseiten der Musikhochschulen öffentlich zugänglichen Lehrendenverzeichnisse ausgewertet worden. In einem nächsten Schritt nahm die Bundeskonferenz der Frauen- und Gleichstellungsbeauftragten an Hochschulen (bukof) und deren Kommission »Künstlerische Hochschulen « die Initiative auf. Um die Genauigkeit der Zahlen zu erhöhen und um die Hochschulen in den Prozess einzubinden, wurden im Mai 2019 die 23 deutschen Musikhochschulen angeschrieben und gebeten, die Zahlen abzugleichen und gegebenenfalls zu korrigieren. Von 23 Hochschulen antworteten zehn im Rahmen der genannten Frist und übermittelten korrigierte Zahlen, die in die Statistik eingepflegt wurden.

Aus der Erhebung, die auf der Webseite von klangzeitort abrufbar ist, ${ }^{1}$ ergeben sich nach jetzigem Stand folgende Zahlen: Bundesweit liegt der Anteil von Frauen an Professuren, akademischem Mittelbau und bei den Lehrbeauftragten an künstlerischen Hochschulen im Bereich Komposition bei 14,5\%, in dem kleineren Fach der elektroakustischen und medialen Komposition (Elektronische Komposition, Medienkomposition, Filmkomposition, Musikinformatik) ist die Situation mit $10 \%$ Frauenanteil noch weiter zugespitzt, in dem größeren Fachgebiet Musiktheorie mit 20,9\% wiederum etwas besser, wenn auch weit entfernt von einem ausgewogenen Verhältnis. Fasst man die Ergebnisse Komposition, Elektroakustische Komposition, Musiktheorie und verwandte Fächer (Neue Musik, Ensembleleitung, Musikübertragung, Musikinformatik u. a.) zusammen, ergibt sich ein Frauenanteil von 17,3 \% bei den Lehrenden in diesen Bereichen.

Es ist wichtig, im Blick zu behalten, dass diese Ergebnisse nur vorläufig sind: Wie bei jeder Statistik beruhen die Zahlen einerseits auf empirischen Daten, andererseits ist die Auswahl und vor allem eine systematische Deutung dieser Angaben nicht leicht vorzu-

1 http://klangzeitort.de/wp-content/uploads/2020/02/klangzeitort_Ergebnisse-Genderverteilung-gesamtaktualisiert-korrigiert-03_200901.pdf (15.5.2020). 
nehmen. Die teilweise komplizierte Zuordnung zu den Lehrgebieten wie auch zu den Statusgruppen erschweren die Einordnung der Befunde: Bei den Lehrgebieten sind die Übergänge oft fließend, so gab es in Komposition und Musiktheorie historisch immer schon Überschneidungen der Lehrpersonen, ebenso zwischen Komposition und elektronischer Komposition. Im Lehrgebiet der elektronischen Musik gibt es Schnittmengen mit den Fachgebieten Filmmusik, Tonmeisterausbildung und verwandten Studiengangsprofilen. Gast- und Honorarprofessuren sowie akademische Mitarbeiter*innen haben häufig befristete Verträge, was nicht berücksichtigt werden konnte, ebenso wenig wie das Ausmaß der Stellenanteile (in allen Statusgruppen). Hinzu kommt, dass die Webseiten der Hochschulen nicht immer auf dem aktuellen Stand sind.

Andererseits ist es ebenso wichtig, zu betonen, dass es nicht Ziel dieser Erhebung war, sich mit wissenschaftlichen Studien zu messen; sie soll in erster Linie dazu dienen, das Augenmerk auf einen grundlegenden Missstand an Hochschulen mit weitreichenden Folgen für das Musikleben zu richten. Statistische Herangehensweisen sind erhellend, da sie vorliegende Daten prägnant zusammenfassen und als Grundlage für weitere Forschung oder Handlungen zur Verfügung stellen. Die in der Recherche zusammengestellten Zahlen weisen ein so eindeutiges - um nicht zu sagen erschreckendes und ernüchterndes Missverhältnis der Genderverteilung bei Lehrenden auf, dass einzelne Verschiebungen in den Rubriken nichts an der Gesamtaussage ändern würden: Ganz konkret bedeuten die Zahlen, dass einzeln betrachtet an nicht wenigen Hochschulen in den Lehrgebieten Komposition und elektroakustische Komposition ausschließlich Männer lehren.

\section{ZUM AKTUELLEN DISKURS ÜBER GENDER UND DIVERSITÄT IN DER ZEITGENÖS- SISCHEN MUSIK UND KOMPOSITION}

Ausgangspunkt dieser Recherche waren zwei Entwicklungen: Zum einen findet seit einigen Jahren im zeitgenössischen Musikleben ein Aufbruch statt. ${ }^{2}$ Die geringe Präsenz von Frauen vor allem als Komponistinnen und kreative Musikschaffende wird thematisiert, und Jurys, Kulturpolitiker*innen und Kurator*innen, vor allem die Musikschaffenden selbst, setzen sich für eine gerechtere Genderverteilung und mehr Diversität ein. Intendant*innen programmieren mehr Frauen in ihren Festivals und Konzertformaten und bekunden, dies in Zukunft noch auszuweiten. Bundesweite Förderinstrumente (z. B. der Musikfonds e. $V$.) werden von politischer Seite angehalten, genderbezogene Kriterien bei Entscheidungen zu berücksichtigen. Bei den Darmstädter Ferienkursen für Neue Musik führte die 2016 durch GRID (Gender Relations in Darmstadt) und GRINM (Gender Relations in New Music) ausgelöste Diskussion 2018 zu einer Quote bei den Teilnehmer*innen der Darmstädter Ferienkurse sowie einer weitgehend paritätischen Verteilung bei den Lehrenden und aufgeführten Komponist*innen. Die Bundeskulturstiftung finanzierte das Defragmentation Programm ${ }^{3}$ der einflussreichsten zeitgenössischen Musikfestivals (Darmstädter Ferienkurse, Donaueschinger Musiktage, MaerzMusik und Ultima Festi-

3 Defragmentation - Curating Contemporary Music ist ein Forschungsprojekt mit dem Ziel, die aktuell in vielen Sparten geführten Diskurse um Gender und Diversity, Dekolonisierung und technologischen Wandel nachhaltig in Institutionen der Neuen Musik zu verankern und kuratorische Praktiken in diesem Bereich zu diskutieren. (https://internationales-musikinstitut.de/de/ferienkurse/defragmentation, 15.5.2020) 
val Oslo). Bereits 2014 begann das Festival Heroines of Sound, elektronische und elektroakustische Musik von Frauen und non binary composers genreübergreifend - aus den Bereichen der sogenannten sakademischen nannten populären Electronica-Szene - zu präsentieren, und erreichte damit den Zuspruch eines großen Publikums. ${ }^{4}$

Zum anderen fanden, wiederum beeinflusst von den Entwicklungen im öffentlichen $\mathrm{Mu}$ sikleben, an der UdK Berlin und im Rahmen von klangzeitort vermehrt Veranstaltungen zur Genderthematik statt. Bereits 2014 wurde ein Gespräch mit der Kulturwissenschaftlerin Christina von Braun über die kulturhistorischen und soziologischen Hintergründe und die möglichen Ursachen für die Unterrepräsentanz von Frauen in der Komposition und in der Neuen Musik geführt und veröffentlicht. ${ }^{5}$ In diesem Zusammenhang wurde auch eine kleine informelle Studie zur aktuellen Präsenz (rückblickend auf drei Jahre) von Frauen im zeitgenössischen Musikleben erstellt: als Komponistinnen auf Festivals und Konzertreihen, bei der GEMA, als Mitglieder der Akademie der Künste Berlin und als Lehrende für Komposition an deutschen Musikhochschulen. Die ermittelten Zahlen lagen um die smagischen dreizehn Prozentı, ganz ähnlich den von der Initiative female:pressure ausgewerteten Statistiken in Bezug auf elektronische >Nicht-U «-Musik. ${ }^{6}$ Die Veranstaltungsreihe FEM ${ }^{*} \_$MUSIC ${ }^{*}$ startete im Herbst 2017 mit FEM*_MUSIC*_FOCUS, einer Diskussionsreihe mit auswärtigen Gästen (Kuratorinnen, Komponistinnen, Instrumentalistinnen). Es folgten $F E M^{*}{ }_{-} M U S I C^{*}{ }_{-} A R C H I V E$, FEM ${ }^{*} M U S I C^{*}{ }_{-} P L A Y$ und $F E M^{*}{ }_{-} M U S I C^{*}{ }_{-} R E A D .^{7}$ Die gesamte Reihe mit Vorträgen, Seminaren und Lektüresessions wurde von Studierenden und Lehrenden gemeinsam vorbereitet und durchgeführt.

In all diesen Zusammenhängen und Diskussionen wurde immer wieder die immanente Unterrepräsentation von weiblichen Lehrenden vor allem im Fächerkomplex `Komposition ‘ an deutschen Musikhochschulen erwähnt. Die Recherche hat diese Beobachtung bestätigt und liefert dazu eine erste statistische Grundlage.

\section{VORBILDER UND NACHWUCHS IN AUSBILDUNG UND LEHRE}

Die Ausbildung an einer Musikhochschule steht am Beginn der professionellen Laufbahn von Komponist*innen, Instrumentalist*innen, Pädagog*innen und Wissenschaftler*innen. Ungeachtet der aktuellen Debatten in der Gesellschaft und in den verschiedenen Szenen und Communities des Kulturlebens, in das die Absolvent*innen nach ihrem Studium entlassen werden, finden sie an den Hochschulen in den Bereichen Komposition und Musiktheorie momentan noch eine Situation vor, in der überwiegend männliche Lehrende unterrichten. Anders als bei den Entscheidungsträgern im öffentlichen Musikleben und in der freien Szene gibt es bei den die Forschung und Lehre prägenden Positionen in den Hochschulen wenig Fluktuation: Professuren werden nach wie vor meistens unbefristet besetzt. Dies ist einerseits ein Grund dafür, dass Frauen entgegen anderslautender Zielsetzungen der Politik und Hochschulleitungen in diesen Positionen weiterhin unterrepräsentiert sind, andererseits zeigt dies auch die Wichtigkeit, Professuren zukünftig vermehrt

$4 \quad$ Vgl. Sanio/Wackernagel 2019.

5 Braun 2014.

6 Vgl. die Webseite female:pessure: http://www.femalepressure.net (15.5.2020).

$7 \quad$ Krafeld u. a. 2019; Rustler 2019. 
mit Frauen zu besetzen, da es sich hier um eine entscheidende Mittler- und Vorbildfunktion für die nächste Generation handelt.

Angesichts des augenfälligen Missverhältnisses muss es für die Hochschulen Ziel und Aufgabe sein, mögliche Benachteiligungsmechanismen in den künstlerischen, künstlerisch-pädagogischen und -wissenschaftlichen Fächern zu analysieren und Wege zu einer Veränderung aufzuzeigen. Hierbei geht es in erster Linie nicht nur (aber auch!) um die akademische Laufbahn von Komponistinnen, Klangkünstlerinnen und Musiktheoretikerinnen: Viel wichtiger noch ist die Bedeutung, die die Präsenz von weiblichen Lehrenden für den Nachwuchs hat. Geschlechtsspezifische Ausgrenzung ist ein Prozess, der über tradierte soziale Strukturen und Codes funktioniert, meist unbewusst wirkt und sich fortsetzt. Da die Zusammensetzung von Kollegien häufig undurchsichtigen Prozessen folgt, sollten Transparenz und Monitoring nicht länger als Angriff auf die Freiheit der Lehre oder der Künste abgetan werden. Umgekehrt lässt sich beobachten, dass eine stärkere Präsenz von Frauen auf institutioneller Ebene als Multiplikator wirkt und über Vorbilder einen weiteren Zuwachs von Frauen in allen Tätigkeitsbereichen initiieren kann. Vielfach wirken unbewusste Mechanismen über Kommunikationswege, die oft unartikuliert (nichtsprachlich vermittelt) und unreflektiert ablaufen.

"Passe ich dazu? Traue ich mir das zu? - Heutzutage spielt wahrscheinlich weniger das fehlende Zutrauen in die eigenen Fähigkeiten eine Rolle, als vielmehr der Zweifel daran, ob man sich in dem sozialen Gefüge, dem akademischen und künstlerischen Betrieb durchsetzen und dort selbstverständlich einen Platz beanspruchen kann. Von Seiten der Entscheidungsträger*innen (nach wie vor meistens männlich) findet häufig gar keine bewusste Ausgrenzung statt, vielmehr handelt es sich hier um das bekannte Muster, dass man sich selbst stärkt, indem man diejenigen fördert, die einem ähnlich sind, im Sinne des Konzepts der `Homosozialität ‘. ${ }^{8}$

Ein zentrale Rolle spielt hier zudem die Vereinbarkeit von Familie und Beruf: Denn prekäre oder befristete Beschäftigungsverhältnisse und der Erwartungsdruck einer hohen örtlichen Flexibilität, wie sie in der Hochschullehre vor allem durch den im Vergleich zu Universitäten überproportional hohen Anteil von Lehraufträgen weit verbreitet sind, führen zumindest der Tendenz nach zu einer strukturellen und systemimmanenten Benachteiligung insbesondere von Frauen in der Familiengründungsphase in ihrer akademischen Laufbahn. ${ }^{9}$

Immer noch studieren weniger Frauen Komposition als Männer (an deutschen Musikhochschulen 2014: ca. $30 \%) .{ }^{10}$ Dass der berüchtigte Karriere-Knick nicht erst zu einem späteren Zeitpunkt stattfindet, sondern dass schon ganz zu Beginn der Ausbildung ein Ungleichgewicht besteht, unterscheidet die hier im Mittelpunkt stehenden Fächer wesentlich von anderen künstlerischen Disziplinen. Vor allem junge Absolvent*innen, die gerade die Hochschulausbildung durchlaufen haben, weisen darauf hin, wie wichtig es sei, dass mehr weibliche und non-binary Studierende zu diesem Studium ermutigt werden. Hier spielt neben weiblichen Vorbildern aus dem außeruniversitären Musikschaffen, denen die zukünftigen Studierenden im Musik- und Instrumentalunterricht sowie im Kultur-

8 Zum Konzept der ’Homosozialität` in der soziologischen Genderforschung siehe Meuser 2010, 430; siehe auch den Überblick zu organisationssoziologischen Ansätzen in Aulenbacher/Riegraf 2010.

9 Siehe auch den Bericht des Bundesministeriums für Bildung und Forschung zur Familienfreundlichkeit an deutschen Hochschulen (2013): https://www.bmbf.de/upload_filestore/pub/familienfreundlichkeit_ an_deutschen_hochschulen.pdf (15.5.2020). 
und Medienleben begegnen, auch das Außenbild einer Hochschule eine wichtige Rolle, also ob dieses auch durch weibliche und diverse Lehrende und Studierende geprägt ist.

Ein weiterer wichtiger Schlüssel sind die Aufnahmeprüfungen, auch im Hinblick auf weitere Aspekte von Diversität wie z. B. der soziokulturellen Herkunft und Identität. In Aufnahmeprüfungen werden besondere Qualitäten in Bezug auf - bereits zuvor erworbenes, also vom Umfeld der jungen Menschen abhängiges - handwerkliches Können und künstlerische Begabung gefordert. Gerade im künstlerischen Bereich ist `Qualitätı jedoch ein nicht immer eindeutig definierbarer, vor allem aber immer neu zu verhandelnder Begriff. Qualitätsvorstellungen haben mit historisch gewachsenen Bildungsstandards und mit dem persönlichen Bildungshintergrund der Prüfenden zu tun. Die eigenen Maßstäbe sehr persönlich vermitteln zu können, ist eine Stärke der künstlerischen Ausbildungskonstellation aufgrund des engen Lehrer*innen-Schüler*innen-Verhältnisses, aber auch ihr Manko, wenn die eigenen Maßstäbe zu sehr in anderen gesucht und auf andere übertragen werden. Dies bedeutet, dass die institutionellen Strukturen und die akademische Selbstverwaltung ein Reproduzieren der existierenden Kriterien begünstigen.

\section{WAS TUN?}

Die Erhebung der Zahlen und die Aufforderung an die Hochschulen, diese zu überprüfen und zu ergänzen, hatten zum Ziel, dass Präsidien, Dekanate und Hochschulgremien sich zu dieser Sachlage positionieren. Die Statistik sollte eine breite Debatte darüber auslösen, welche hochschulspezifischen Maßnahmen dazu dienen können, gendergerechte Bedingungen zu schaffen und Chancengleichheit zu fördern. Bestehende Maßnahmen, von denen man den Eindruck hat, dass sie oft nur auf dem Papier stehen, sollten wieder mehr Durchschlagskraft erhalten - immerhin handelt es sich bei Gleichstellung um einen gesetzlichen Auftrag.

Konkrete Vorschläge wären:

- Einhaltung bereits vorliegender Richtlinien und Hochschulgesetze, die der Umsetzung des gesetzlichen Gleichstellungsauftrags dienen, sowie ein unabhängiges Monitoring, wie sich die Zahlen entwickeln und ob gegebenenfalls umgesteuert werden muss, um Zielquoten zu erreichen;

- paritätische Besetzung von Berufungskommissionen, ggf. mit Mitgliedern von außerhalb der betreffenden Hochschule, Erstellen einer hochschulübergreifenden Liste (Pool) mit qualifizierten Frauen aus den jeweiligen Fachrichtungen;

- eine hochschulübergreifende Diskussion darüber, wie Berufungsverfahren im Rahmen der gesetzlichen Regelungen transparenter gestaltet werden können, z. B. im Hinblick auf Statistiken zum Gender von Bewerber*innen, eingeladenen Bewerber*innen, Listenplätze, Neuberufungen;

- konsequente Entwicklung belastbarer Instrumente, damit sich mehr Frauen auf Professuren und andere Positionen bewerben; Hinterfragen von Mechanismen in Berufungsverfahren, die dazu führen, dass Frauen den Eindruck gewinnen, sie würden lediglich vorgeführt; stattdessen Entwicklung von professionell geführten Berufungsverfahren, deren Planung und Durchführung sich an erfolgreicher Gleichstellungspolitik (১best practice «) im künstlerischen oder akademischen Bereich im In- und Ausland oder z. B. an den Besetzungsverfahren für Führungskräfte in Bundesbehörden infolge des Bundesgleichstellungsgesetzes (BGleiG) orientiert; 
- strukturierte gendersensible Verfahren und Transparenz auch für die Besetzung von Stellen und Positionen bei Gastprofessor*innen, akademischen Mitarbeiter*innen und Lehrbeauftragten;

- Einführung des 'Kaskadenmodells`, nach dem der Frauenanteil jeder akademischen Karrierestufe mindestens so hoch sein muss, wie derjenige der direkt darunterliegenden Qualifizierungsstufe;

- Maßnahmen zur Erhöhung der Vereinbarkeit von Familie und Beruf, etwa durch mehr Perspektiven auf entfristete Stellen auch jenseits der Lebenszeitprofessur;

- Maßnahmen, die gezielt auf eine Erhöhung des Anteils weiblicher Studierender und mehr Diversität in den genannten Fächern hinwirken, z. B. durch die Entwicklung von Aufnahmeverfahren und Kriterien, die noch differenzierter die künstlerische Eignung und das persönliche Potential ermitteln und nicht nur erworbenes Vorwissen abfragen;

- Fachaustausch mit Kolleginnen und Kollegen, z. B. im Hinblick auf die Einbeziehung von Komponistinnen und Musikerinnen aller Epochen in die Lehre und den `Kanon (Überarbeitung von Curricula und Repertoire im Fachunterricht, Bereitstellung von Partituren, Aufnahmen und weiterer Materialien in Hochschulbibliotheken).

Darüber hinaus sollten hochschulübergreifende und außerhochschulische Netzwerke aktiviert werden, die von ihrer Seite aus Forderungen an die Hochschulen stellen und beispielsweise Berufungsverfahren und Abläufe bei Stellenbesetzungen beraten, begleiten und kommentieren.

Das Musikleben, der Musikbetrieb braucht und will Diversität, und bei den Hochschulen sollte es anfangen.

\section{Literatur}

Aulenbacher, Brigitte/ Birgit Riegraf (2010), „Geschlechterdifferenzen und -ungleichheiten in Organisationen«, in: Soziologische Geschlechterforschung. Eine Einführung, hg. von Brigitte Aulenbacher, Michael Meuser und Birgit Riegraf, Wiesbaden: VS Verlag für Sozialwissenschaften, 157-171.

Braun, Christina von (2014), „Wer ist hier der Herr im Haus? Oder: Die magischen $13 \%$. Christina von Braun im Gespräch mit Wolfgang Heiniger, Kirsten Reese, Cornelia Schmitz, Iris ter Schiphorst", in: kzo-Programmleporello des Instituts für Neue Musik der Universität der Künste April/Mai, Juni, Juli 2014. http://alt.klangzeitort.de/index.php? page=neuemusikimdiskurs.html\&id=3\#document (15.5.2020)

Krafeld, Merle / Lucien Danzeisen / Rosanna Lovell / Evelyn Saylor / Stellan Veloce (2019), FEM*_MUSIC_*, Berlin: Universität der Künste.

Meuser, Michael (2010), »Junge Männer: Aneignung und Reproduktion von Männlichkeit", in: Handbuch Frauen- und Geschlechterforschung. Theorie, Methoden, Empirie, 3. Auflage, hg. von Ruth Becker und Beate Kortendiek, Wiesbaden: VS Verlag für Sozialwissenschaften, 428-435.

Reese, Kirsten (2019), „Was sich geändert hat. Ein Blick auf Gendergerechtigkeit in der zeitgenössischen `Neuen Musik«", in: Heroines of Sound. Feminismus und Gender in elektronischer Musik, hg. von Sabine Sanio und Bettina Wackernagel, Hofheim: Wolke, 76-83. 
Rustler, Katharina / FEM*_MUSIC*_ (2019), „Feminismus ist keine Betriebsstörung «. https://www.udk-berlin.de/universitaet/gleichstellungspolitik/akteurin-sein/divaversityof-arts/feminismus-ist-keine-betriebsstoerung (15.5.2020)

Sanio, Sabine / Bettina Wackernagel (Hg.) (2019), Heroines of Sound. Feminismus und Gender in elektronischer Musik, Hofheim: Wolke.

Schulz, Gabriele / Carolin Ries / Olaf Zimmermann (2016), Frauen in Kultur und Medien. Ein Überblick über aktuelle Tendenzen Entwicklungen und Lösungsvorschläge, Berlin: Deutscher Kulturrat e. V. https://www.kulturrat.de/wp-content/uploads/2016/12/Frauenin-Kultur-und-Medien.pdf (15.5.2020)

Kletschke, Irene / Reese, Kirsten (2020): Genderverteilung der Lehrenden in den Fächern Komposition, Elektroakustische Komposition und Musiktheorie an deutschen Hochschulen. Eine statistische Recherche [Gender Balance of Teachers in Composition, Electroacoustic Composition, and Music Theory in German Universities. A Statistical Study]. ZGMTH 17/1, 147-153.

https://doi.org/10.31751/1042

(C) 2020 Irene Kletschke (kletschk@udk-berlin.de), Kirsten Reese (kireese@udk-berlin.de) Universität der Künste Berlin [Berlin University of Arts], Universität der Künste Berlin [Berlin University of Arts]

Dieser Text erscheint im Open Access und ist lizenziert unter einer Creative Commons Namensnennung 4.0 International Lizenz.

This is an open access article licensed under a

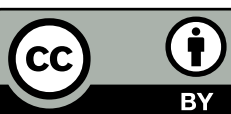

Creative Commons Attribution 4.0 International License.

eingereicht / submitted: 21/04/2020

angenommen / accepted: 22/04/2020

veröffentlicht / first published: 15/06/2020

zuletzt geändert / last updated: 15/06/2020 